\title{
Uso de videolaringoscopio Glidescope para manejo de vía aérea difícil en paciente con tumor de cuerdas vocales
}

\author{
José Raúl Salinas Rojas, ${ }^{1}$ José Granados Barrientos, ${ }^{1}$ Armando Valderrábano López, ${ }^{1}$ \\ Masao Kume, ${ }^{2}$ Alfonso Ramírez Gorostiza ${ }^{2}$
}

\section{Resumen}

Introducción: Los pacientes sometidos a procedimientos quirúrgicos de vía aérea superior constituyen un grupo de alto riesgo, con alta probabilidad de complicaciones ventilatorias y respiratorias. La American Society of Anesthesiology intenta facilitar la probabilidad de éxito y disminuir riesgos a través de "guías de manejo de vía aérea difícil". Caso clínico: Paciente masculino de 68 años de edad con diagnóstico de tumor de cuerdas vocales, programado para resección de tumor por microcirugía con láser; índice predictivo de intubación difícil: 9 (alto). Se realiza intubación con videolaringoscopio Glidescope; se observa obstrucción de vía aérea del $90 \%$; se coloca tubo endotraqueal sin complicaciones, sin lesiones ni sangrado de la masa tumoral. Conclusiones: El uso de equipo de intubación videoasistida Glidescope nos proporciona una visión panorámica de la glotis y esto facilita de manera importante el manejo de la vía aérea difícil.

Palabras clave: Vía aérea difícil, tumor de cuerdas vocales, videolaringoscopio.

\section{INTRODUCCIÓN}

El inadecuado manejo de vía aérea difícil sigue siendo causa del $50 \%$ de las complicaciones quirúrgicas relacionadas con la anestesia; los principales eventos adversos asociados son: fracturas dentales, trauma en cavidad oral, trauma de faringe o laringe, alteraciones cardiopulmonares, daño cerebral,

\footnotetext{
${ }^{1}$ Médico Anestesiólogo, Hospital Ángeles Pedregal. Facultad Mexicana de Medicina - Universidad La Salle.

${ }^{2}$ Médico Otorrinolaringólogo, Hospital Ángeles Pedregal. Fundación Mexicana de la Voz.
}

Correspondencia:

José Raúl Salinas Rojas

Correo electrónico: chelo_salinasjr@hotmail.com

Aceptado: 25-05-2017.

Este artículo puede ser consultado en versión completa en http:// www.medigraphic.com/actamedica

\begin{abstract}
Summary
Introduction: Patients undergoing upper airway surgical procedures constitute a high-risk group, with a high probability of respiratory complications. The American Society of Anesthesiology attempts to facilitate the likelihood of success and decrease risks through "difficult airway management guides". Case report: A 68-year-old male patient with a vocal cord tumor diagnosis, programmed for resection of a tumor by microsurgery with laser, predictive index of difficult intubation 9 (high), intubation with videolaryngoscope Glidescope, airway obstruction of $90 \%$, is placed endotracheal tube without complications, without injury or bleeding of the tumor mass. Conclusions: The use of Glidescope assisted video intubation equipment gives us a panoramic view of the glottis and this significantly facilitates difficult airway management.
\end{abstract}

Key words: Difficult airway, vocal cord tumor, videolaryngoscope.

incluso la muerte. Los motivos de este problema podrían resumirse en una deficiente formación para el manejo de vía aérea difícil, falta de destreza en técnicas alternativas y desconocimiento de algoritmos de actuación necesarios ante estas situaciones. Tal déficit de entrenamiento radica en la baja incidencia de pacientes con vía aérea difícil y la dificultad para su predicción. ${ }^{1}$

Pacientes sometidos a procedimientos quirúrgicos de vía aérea superior constituyen un grupo de alto riesgo; esto implica trabajar en un área muy limitada, de difícil acceso, y una alta probabilidad de complicaciones ventilatorias y respiratorias; para minimizar estos riesgos es relevante conocer a profundidad la técnica quirúrgica planeada.

El médico anestesiólogo, siendo el mayor responsable del manejo de la vía aérea antes, durante y después del evento quirúrgico, ${ }^{2}$ debe realizar una evaluación preoperatoria completa para establecer antecedentes patológicos y quirúrgicos, en especial cirugías previas de cabeza y 
cuello, un examen físico que determine las condiciones generales del paciente y una revisión exhaustiva de la vía aérea. Se deberá contemplar siempre que las dificultades para ventilar o intubar pueden agravarse tras la inducción anestésica y la relajación muscular. ${ }^{3}$

La American Society of Anesthesiology publica "Practice Guidelines for Management of the Difficult Airway", con el objeto de facilitar la probabilidad de éxito y disminuir los riesgos. El plan de manejo dependerá siempre de la condición del paciente, la habilidad y juicio del anestesiólogo y la disponibilidad de recursos tecnológicos. ${ }^{4}$

Se han implementado varios índices y mediciones de predictores de vía aérea difícil, entre los cuales están: Mallampati, distancia esternomentoniana, distancia tiromentoniana, distancia interincisivos, apertura bucal y protrusión mandibular. ${ }^{5}$ Existen otros factores que pueden influir en el manejo de la vía aérea, como son: sobrepeso, diámetro del cuello, estado de las piezas dentales y edentación, macroglosia, neoplasias obstructivas. ${ }^{6}$ La escala de Cormack-Lehane valora el grado de dificultad para la intubación después de realizar una laringoscopia directa, según las estructuras anatómicas que se visualicen, siendo el grado tres y cuatro un índice predictivo de dificultad durante la intubación.

Las nuevas técnicas de intubación facilitan la visualización de la glotis mediante sistemas de transmisión de la imagen por video o fibra óptica. La intubación mediante estos dispositivos es subjetivamente más fácil que con la laringoscopia directa, aunque su superioridad en cuanto a rapidez, éxito en la intubación y número de intentos está por demostrarse; esto podría deberse al conocimiento de la técnica, entrenamiento adecuado, habilidad y experiencia del anestesiólogo. ${ }^{7}$

Un videolaringoscopio tiene incorporado un sensor electrónico de imagen en el tercio distal de la hoja, transmitiendo la imagen digital a una pantalla LCD. Junto a este sensor está inserta una fuente de luz LED (Light Emitting Diode) que proporciona mayor intensidad lumínica que una luz fría convencional y con una irradiación espectral más cercana al ojo humano. ${ }^{8}$

El Glidescope (Saturn Biomedical System, Inc., Burnaby, British Columbia, Canadá) desarrollado por el Dr. John A. Paley y comercializado desde el 2001 en Canadá, es el videolaringoscopio con mayor difusión en el mundo y ha merecido muchas publicaciones en los últimos años. Fue diseñado especialmente para el manejo de la vía aérea difícil y en condiciones especiales como inmovilidad cervical, obesidad mórbida, paciente quemado, trauma facial y tumores de laringe. ${ }^{9}$

\section{PRESENTACIÓN DEL CASO CLÍNICO}

Paciente masculino de 68 años de edad diagnosticado con tumor de cuerdas vocales. Se programó para re- sección de masa tumoral por microcirugía de laringe con láser.

Antecedentes patológicos de importancia: diabetes mellitus tipo 2 desde hace 15 años en tratamiento con metformina $850 \mathrm{mg} /$ día vía oral; antecedentes quirúrgicos: apendicetomía, microcirugía de laringe en dos ocasiones, antecedentes de vía aérea difícil. Al examen físico, clínicamente normal, con signos vitales: frecuencia cardiaca 72 latidos x min, presión arterial 120/75 mmHg, $\mathrm{SpO}_{2} 80 \%$ al aire ambiente, frecuencia respiratoria 18 respiraciones $x$ min. Medidas antropométricas: peso, $80 \mathrm{~kg}$; talla, $1.70 \mathrm{~m}$.

Valoración de vía aérea: Mallampati III, distancia esternomentoniana II, distancia tiromentoniana II, distancia interincisivos I, protrusión mandibular I, índice predictivo de intubación difícil, (IPID) de 9. Cuello corto con movilidad conservada, tráquea central móvil, sin adenomegalias. El paciente se somete a fibroscopia diagnóstica en la cual se observa masa tumoral importante en cuerdas vocales y sinequia de laringe con obstrucción de vía aérea del 90\% (Figura 1).

Exámenes de laboratorio dentro de parámetros normales; electrocardiograma y placa radiográfica de tórax sin datos patológicos aparentes.

Valoración del riesgo anestésico quirúrgico (RAQ) EIIA, riesgo tromboembólico moderado, vía aérea difícil. Plan: sin contraindicaciones para procedimiento quirúrgico, ayuno previo de ocho horas, trombo profilaxis mecánica con medias TED, anestesia general balanceada.

Previa solicitud del equipo de intubación video asistida y fibroscopio flexible en sala, así como del material necesario para manejo de vía aérea quirúrgica y la presencia de todo el equipo quirúrgico en sala, pasa paciente con venoclisis permeable en miembro superior izquierdo, se procede a monitorización de frecuencia cardiaca, electrocardiograma continuo, presión arterial no invasiva, saturación de oxígeno, monitoreo de relajación muscular. Se realiza oxigenación con mascarilla facial durante 10 minutos hasta lograr $\mathrm{SpO}_{2}$ mayor a 95\%; se inicia la inducción anestésica con fentanilo $2 \mathrm{mg} / \mathrm{kg}$, propofol $1.5 \mathrm{mg} / \mathrm{kg}$ y atracurio $0,4 \mathrm{mg} / \mathrm{kg}$; se efectúa laringoscopia con equipo de intubación video asistida (Glidescope) (Figura 2) hoja \#4 y realizando una sutil maniobra se empiezan a identificar las estructuras con mucha dificultad; se evidencian abundantes secreciones y se observa masa tumoral que obstruye casi por completo (90\%) la luz de la vía aérea. Se decide introducir una sonda endotraqueal de diámetro pequeño $(5 \mathrm{~mm})$ con globo, utilizando estilete rígido de ángulo $90^{\circ}$, la cual atraviesa sin producir lesión de masa tumoral con un mínimo sangrado. Una vez hecho esto, se corrobora la correcta intubación con el monitor de capnografía, se fija la sonda endotraqueal casi en la comisura labial de lado izquierdo y se conecta el circuito a ventilación mecánica (Figura 3). Después se realiza la extirpación de masa tumoral, localizada en ambas cuerdas 
vocales, con invasión del espacio paraglótico; se retira pieza quirúrgica para patología y hemostasia de lechos sangrantes; se observa una mejoría absoluta a nivel de la luz de vía aérea; se termina el procedimiento sin incidentes ni complicaciones, con parámetros hemodinámicos y ventilatorios estables.

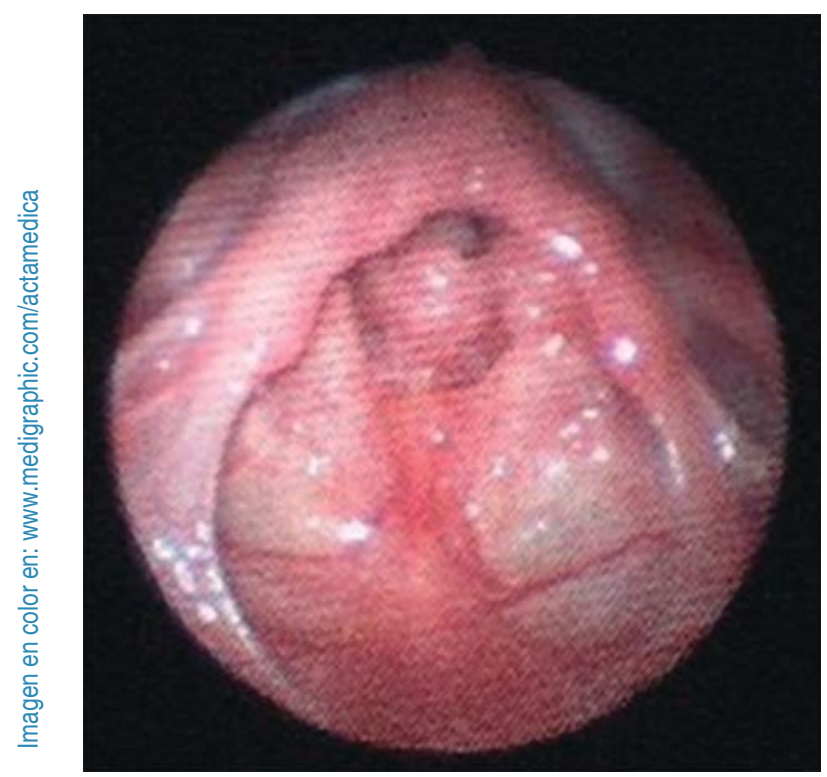

Figura 1. Fibroscopia diagnóstica: se observa obstrucción del $90 \%$ de vía aérea por tumor de cuerdas vocales y laringe.

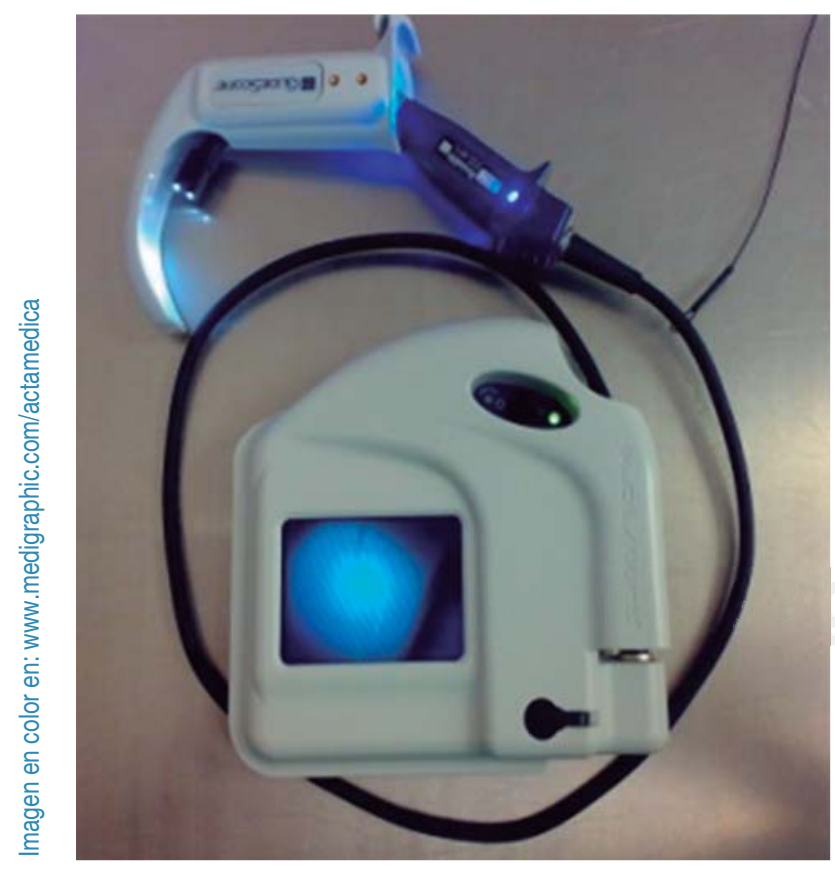

Figura 2. Videolaringoscopio Glidescope.
Se decide emersión de estado anestésico, extubación del paciente, con previa aspiración de secreciones y presencia de ventilación espontánea, se retira cánula endotraqueal y se apoya ventilatoriamente con mascarilla facial y oxígeno, cinco litros por minuto. Pasa a área de recuperación con Aldrete 9/10 $\mathrm{SpO}_{2}$ 95\%.

\section{DISCUSIÓN}

Las dificultades en la predicción de la vía aérea difícil, hacen que uno de cada tres casos no sea previsto en la evaluación preoperatoria. ${ }^{10}$ Muchos protocolos de estudio demuestran que la combinación de pruebas predictivas y la inclusión en estas escalas de los antecedentes de vía aérea difícil, patología asociada a la misma y síntomas de compromiso de la vía aérea (estridor, disnea, disfonía, disfagia, odinofagia) han mejorado significativamente los índices de éxito. ${ }^{11}$

Sin lugar a dudas, el desarrollo de video laringoscopios representa el mayor avance en el manejo de la vía aérea logrado en esta década; los nuevos esquemas facilitan un manejo que anteriormente ocasionaba mayor incertidumbre. $^{12}$

El Glidescope es el videolaringoscopio más utilizado en la actualidad y ya cuenta con una amplia difusión de estudios clínicos y experiencia en el manejo de vía aérea difícil. La principal innovación que ofrece es el tipo de hoja, en forma de J, con un ángulo de $60^{\circ}$. Esta hoja se introduce en la cavidad bucal en la línea media y por su diseño del extremo distal se posiciona fácilmente en la vallécula al completar el giro e introducción. La sonda orotraqueal

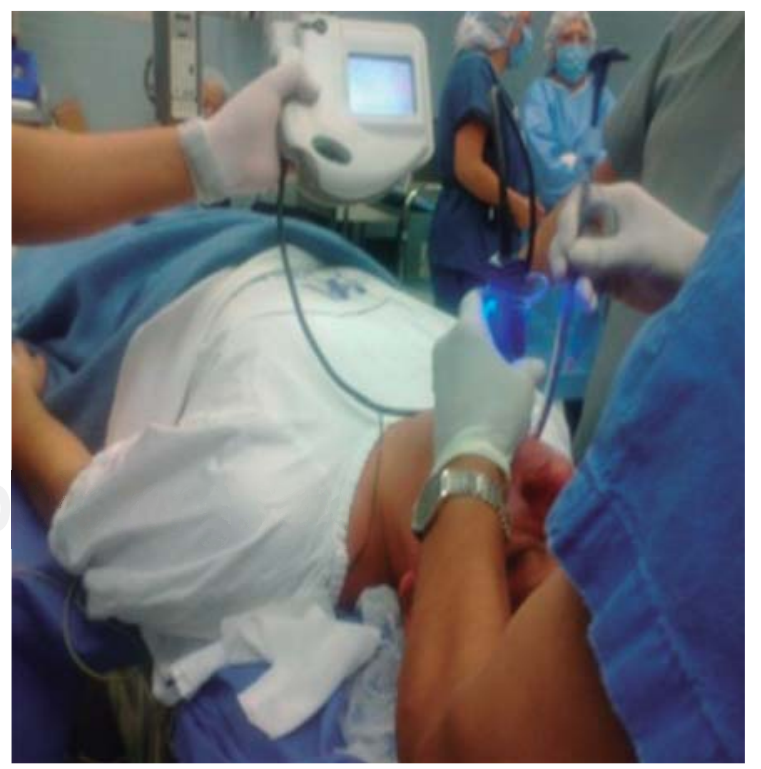

Figura 3. Manejo de vía aérea difícil con Glidescope. 
se introduce en paralelo al Glidescope, facilitada por un estilete con un ángulo de $60^{\circ}$ que viene junto al equipo. ${ }^{13}$

El hecho de tener sensor de imagen en la parte distal de la hoja hace que tengamos una visión panorámica de la glotis, sin necesidad de "alinear los ejes" y en la práctica tener una laringoscopia Cormack-Lehane grado I o II en el $99 \%$ de los casos. Los videolaringoscopios tienen un campo visual de entre 45 y $60^{\circ}$, a diferencia de la visión distante tubular de $15^{\circ}$ que proporciona una laringoscopia clásica. También se ha adaptado un sistema antiempañante para contrarrestar el calor que emiten los diodos. ${ }^{14}$

La experiencia clínica del Glidescope con diversos protocolos y estudios realizados en la población, ha mostrado que casi siempre mejora la visión, pero el éxito de intubación es algo menor, con alrededor del 95\%. Las causas del fracaso se deben a una incompleta curva de aprendizaje, diseño del estilete utilizado, dificultad para avanzar el tubo, empañamiento de la óptica o simplemente una laringoscopia grado IV directa o indirecta. El porcentaje de éxito en el primer intento fluctúa entre 86 y $98 \%$ debido a las mismas variables antes mencionadas. El tiempo de intubación fluctúa entre 30-40 segundos en una vía aérea normal y de 40-80 segundos para una vía aérea difícil. ${ }^{15}$

Se ha estimado que son necesarias por lo menos 30 intubaciones con Glidescope para disminuir significativamente la tasa de fracaso y el tiempo requerido en este procedimiento. ${ }^{16}$

Las complicaciones con el manejo del Glidescope son poco frecuentes; la presión ejercida sobre el maxilar es baja, ya que no se realiza una maniobra de palanca: sólo rotación para optimizar la visión y elevar la glotis. La complicación más frecuente es la perforación del paladar blando o arco palatogloso, que ocurre con la introducción a ciegas del tubo endotraqueal con el estilete rígido. ${ }^{17}$

\section{CONCLUSIONES}

Actualmente no existen pruebas que confirmen o descarten totalmente el diagnóstico de vía aérea difícil. Se deben de seguir tomando en cuenta las mediciones clínicas, los riesgos propios de la patología y las condiciones de cada paciente, para tomar las medidas necesarias y evitar complicaciones.

Es muy importante la comunicación entre cirujano y anestesiólogo para el manejo de estos pacientes, en especial en momentos cruciales como son la intubación y extubación del mismo. Es fundamental que la sala de operaciones cuente con todo el material necesario para el manejo de una vía aérea difícil y las probables complicaciones, como marcan las guías y protocolos descritos, más aún en procedimientos endoscópicos de vía aérea.
El uso de videolaringoscopio es de gran utilidad para la docencia, facilitando la adquisición de destreza. El éxito en el manejo de vía aérea difícil implica no sólo el conocimiento sino también el correcto entrenamiento y la habilidad para la resolución de complicaciones.

El Glidescope facilita de manera importante el manejo de la vía aérea difícil, al permitir una visión panorámica de la laringe, proporcionando laringoscopias grado I en la mayoría de los pacientes; disminuye las maniobras de optimización necesarias para el éxito de la intubación y permite al equipo de anestesiólogos tener visión directa para la colaboración durante la intubación.

Existen estudios que demuestran que con la modificación del ángulo del estilete para la introducción del tubo endotraqueal se facilita la intubación, acortando los tiempos y aumentando la tasa de éxito y la comodidad del médico operador.

Para el manejo alternativo de una vía aérea difícil anticipada el fibrobroncoscopio y la intubación vigil siguen plenamente vigentes.

\section{REFERENCIAS}

1. Fasting $\mathrm{S}$, Gisvold SE. Serious intraoperative problems - a five-year review of 83,844 anesthetics-. Can J Anaesth. 2002; 49 (6): 545553.

2. Cattano D, Killoran PV, lannucci D, Maddukuri V, Altamirano AV, Sridhar $\mathrm{S}$ et al. Anticipation of the difficult airway: preoperative airway assessment, an educational and quality improvement tool. Br J Anaesth. 2013; 111 (2): 276-285.

3. Grünberg G, Bounous A, Prestes I, Amonte G, Illescas L, Ferreira E et al. Evaluación de los métodos predictores de vía aérea dificultosa en pacientes coordinados para procedimientos endoscópicos de vía aérea superior. Anest Analg Reanim. 2006; 21 (1): 11-19.

4. American Society of Anesthesiologists Task Force on Management of the Difficult Airway. Practice guidelines for management of the difficult airway: an updated report by the American Society of Anesthesiologists Task Force on Management of the Difficult Airway. Anesthesiology. 2003; 98 (5): 1269-1277.

5. Khan ZH, Mohammadi M, Rasouli MR, Farrokhnia F, Khan RH. The diagnostic value of the upper lip bite test combined with sternomental distance, thyromental distance, and interincisor distance for prediction of easy laryngoscopy and intubation: a prospective study. Anesth Analg. 2009; 109 (3): 822-824.

6. Wilson ME, Spiegelhalter D, Robertson JA, Lesser P. Predicting difficult intubation. Br J Anaesth. 1988; 61 (2): 211-216.

7. Massó E. Los retos de futuro de la vía aérea en anestesiología. Rev Esp Anestesiol Reanim. 2007; 54: 137-139.

8. Lewis E, Zatman ST, Wilkes AR, Hall JE. Laryngoscope light output. Anaesthesia. 2009; 64 (6): 688-689.

9. Rai MR, Dering A, Verghese C. The Glidescope system: a clinical assessment of performance. Anaesthesia. 2005; 60 (1): 60-64.

10. Bruce RC, McLeod AD, Smith GB. A survey of UK anaesthetic trainee attitudes towards simulator based training experience. Bulletin of the Royal College of Anaesthetists. 2005; 34: 1722-1723.

11. Chang HC, Vásquez YG, Maneiro BJ. Métodos predictivos para una intubación orotraqueal difícil en una población de pacientes del Hospital “Dr. Miguel Pérez Carreño". Rev Ven Anest. 1998; 3: 52-58. 
12. Guzmán OJ. Videolaringoscopios. Rev Chil Anest. 2009; 38 (2): 135-144.

13. Jones PM, Turkstra TP, Armstrong KP, Armstrong PM, Cherry RA, Hoogstra $\mathrm{J}$ et al. Effect of stylet angulation and endotracheal tube camber on time to intubation with the GlideScope. Can J Anaesth. 2007; 54 (1): 21-27.

14. Griesdale DE, Liu D, McKinney J, Choi PT. Glidescope ${ }^{\circledR}$ videolaryngoscopy versus direct laryngoscopy for endotracheal intubation: a systematic review and meta-analysis. Can J Anaesth. 2012; 59 (1): 41-52.
15. Cooper RM, Pacey JA, Bishop MJ, McCluskey SA. Early clinical experience with a new videolaryngoscope (GlideScope) in 728 patients. Can J Anaesth. 2005; 52 (2): 191-198.

16. Mathienson $\mathrm{E}$, JooH, Naik V et al. Learning curve for intubations with the glidescope. Can J Anaesth. 2007; 54: S1.

17. Cooper RM. Complications associated with the use of the GlideScope videolaryngoscope. Can J Anaesth. 2007; 54 (1): 54-57. 